\title{
CEPHALOMETRIC FLOATING NORMS FOR THE MMBP-WITS AND $\beta$ ANGLE AMONG THE CHENNAI POPULATION
}

\author{
Orthodontology \\ Dr. Dhivya \\ Dilipkumar \\ Associate Professor, SRM Kattankulathur Dental College,Tamil Nadu 603203.
}

Dr. Vineeth Kumar PG Student, SRM Kattankulathur Dental College,Tamil Nadu 603203. *Corresponding S* Author

Dr. Krithika. A $\quad$ Senior Lecturer, SRM Kattankulathur Dental College, Tamil Nadu 603203.

Dr. Deenadayalan

Purushothaman

Reader, SRM Kattankulathur Dental College, Tamil Nadu 603203.

Dr. Aravindaksha

Rao

PG Student, SRM Kattankulathur Dental College,Tamil Nadu 603203.

\section{ABSTRACT}

AIM: The purpose of this study was to attain floating norms for the MMBP-Wits and $\beta$ angle in adult Chennai population that can aid in proper diagnosis and treatment planning of different cases. Methods: Lateral cephalograms were acquired from 100 subjects (50 males and 50 females; mean age: $18-25$ years) with pleasant profile and well-balanced and a near-ideal occlusion. Land mark identification and tracings were carried out manually on $0.003^{\prime}$ thickness acetate film. The ANB angle, the $\beta$-angle, the MMBP-Wits were measured. Four Other angular cephalometric parameters which included the SN/PP, SN/MP, SNA, and NSB angles were also measured. Multiple regression models were used to quantify the correlation of the ANB angle, MMBP-Wits and $\beta$ angle with each other and also with four other angular cephalometric parameters mentioned above. RESULTS: All the three cephalometric parameters of antero-posterior maxillary-mandibular relationship had considerable association with the explanatory variables with $\beta 2$ range from 0.183 to 0.212 for the ANB angle and the MMBP-Wits, respectively. The ANB angle was considerably related with all the explanatory variables. In particular, the NSBa, SNA, and SN/MP angles were certainly associated with the ANB angle, whereas the SN/PP angle was inversely associated. On the contrary, both the $\beta$ angle and MMBP-Wits were considerably associated with SNA and SN/MP angles. These explanatory variables showed positive and inverse association with the MMBP-Wits and the $\beta$ angle. CONCLUSION: (i) The ANB angle is prone to have considerably more geometrical distortion as compared to the MMBP-Wits and the $\beta$ angle. (ii) Floating norms have been given to individualize the reference values for both the MMBP-Wits and $\beta$ angle.

\section{KEYWORDS}

ANB angle, Cephalometric analysis, $\beta$ angle, MMBP-Wits

\section{INTRODUCTION}

A precise measurement of the sagittal jaw relationship is essential to plan any orthodontic treatment [2-4]. Linear and angular measurements have been incorporated by researchers into various cephalometric analysis in order to obtain an accurate method for the assessment of sagittal base discrepancy. This was done in order to benefit the clinician to diagnose the skeletal class and thereby formulate the best suitable treatment plan.

ANB angle is one of the most commonly used index to measure sagittal jaw relationship, [2, 3] it is defined as "an angle that denotes the relative position of maxilla and mandible to each other". However, it still poses dissimilarity between the measured angle and the actual skeletal jaw relationship that has been observed [4-7]. The measurement of Wits appraisal on the functional occlusal plane, a second commonly used measurement [3], had been introduced to overcome the shortcomings associated with ANB angle. While Wits appraisal related points A and B to the occlusal plane, has its own limitations which led to the difficulty in the interpreting the cant of this plane [8]

Recently, number of indices have been introduced with the aim to overcome the drawbacks of the indices mentioned above. They were: The Maxillary-Mandibular plane angle bisector (MMBP-Wits) [10] and the $\beta$-angle [9]. C.Y. Baik et.al introduced $\beta$-angle and this angle is defined as "the angle formed between the perpendicular line from point A to the CA-B line and the A-B line" [9]. J. Hall-Scottand was the founder of MMBP-Wits and is defined as "the distance between the perpendicular projection of $\mathrm{A}$ and $\mathrm{B}$ ( $\mathrm{Ap}$ and $\mathrm{Bp}$, resp.) on the bisector of the PP/MP angle"' $[9,10]$

The authors stated that these indices would endure minimal geometrical distortion from facial divergence or jaw rotation in contrast to other indices $[9,10]$. Despite the claim that these two indices could show superior results on cephalometric measurements, future research needs to be done on this aspect and documented in literature.
Thus, the aim of this study was to attain floating norms for the MMBPWits and $\beta$ angle when in comparison with the conventional ANB angle, in relation to facial divergence, in order to acquire individualized cephalometric norms associated with each facial type.

\section{MATERIALSAND METHODOLOGY:}

\section{Study Population and Design:}

This study included subjects who had reported to the Department of Orthodontics and Dentofacial Orthopedics in SRM Kattankulathur Dental College and Hospital seeking orthodontic treatment for minimal tooth alignment without a prior history of orthodontic treatment. As per routine protocol, signed informed consent was obtained from the patients to release their diagnostic data for scientific purposes, prior to commencement of the treatment. The study was permitted by the Local Ethical Committee of the Institution. Pretreatment clinical records were taken, which included Intra-oral and Extra-oral photographs, diagnostic impressions as well as lateral cephalogram. Subjects with a pleasant profile, well-balanced and a near-ideal occlusion were selected for the study.

\section{Inclusion criteria:}

(I) Patients aged 18 to 25 years

(ii) Absence of extensive dental caries

(iii) Absence of extensive dental restorations

(iv) Absence of anterior craniofacial anomaly

(v) No previous history of trauma, fracture in maxillofacial region.

A whole of 100 subjects ( 50 males and 50 females) were considered in the study (mean age of 18-25 years).

\section{Clinical Recordings:}

A well experienced orthodontist assessed the cases that were to be included in the study. Subject profile was assessed by analyzing the extra-oral photographs with the corresponding lateral cephalogram. A Near ideal occlusion was assessed using Intraoral photographs and stone models. As per the norms of the study, the subject had to have a 
Class I molar relationship with less than $1 / 2$ cusp displacement and a canine relationship with less than $1 / 4$ of cusp displacement [16], normal overbite and overjet. Patients with mild incisor irregularities were also considered.

\section{Cephalometric Analysis:}

All the cephalograms were obtained in centric occlusion with lips in a relaxed position. Land mark identification and tracings were carried out manually on 0.003 ' thickness acetate film. The following landmarks were marked for the cephalometric analysis. Indices of sagittal jaw relationship used in this study were: the ANB angle (Figure 1(a)) [1]; $\beta$-angle (Figure 1(b)) [9], and the MMBP-Wits (Figure 1(c)) [10]. Four angular measurements were included as other cephalometric parameters as follows: Maxillary inclination relative to the Cranial Base (SN/PP angle), Mandibular inclination relative to the Cranial Base (SN/MP angle), Maxillary Prognathism (SNA angle) and Cranial Base angle (NSBa angle) (Figure 1(a)). Lateral Cephalograms were standardized with a magnification factor of $0 \%$.

Figure 1(a): the ANB angle, SN/PP angle, SN/MP angle, SNA angle, NSBa angle.

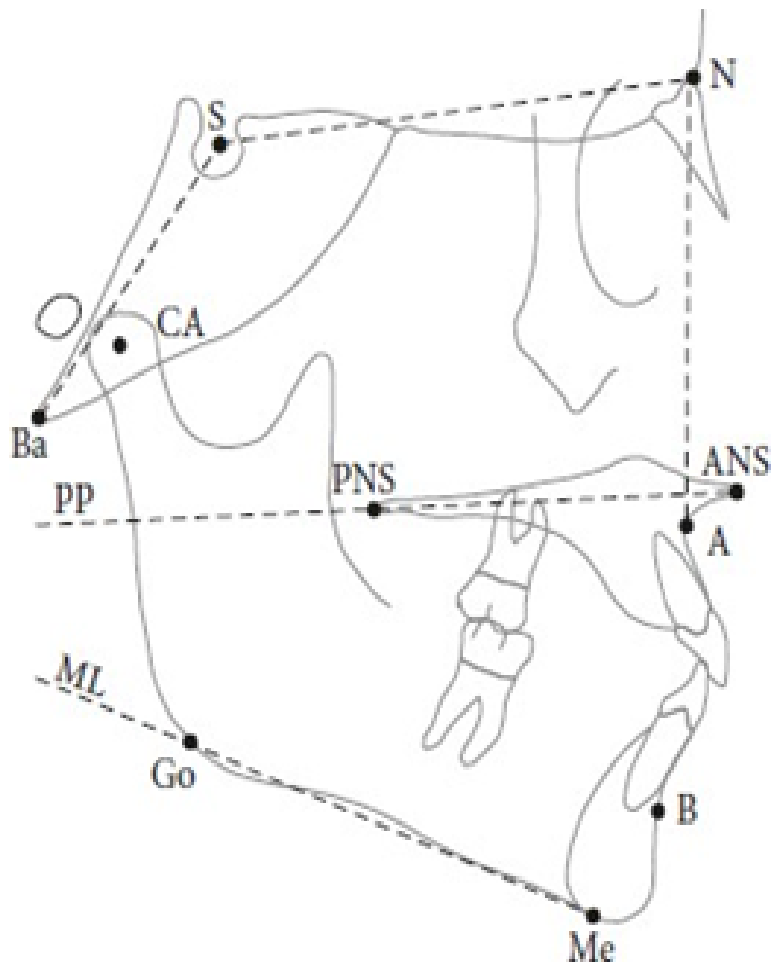

Figure 1(b): $\beta$-angle

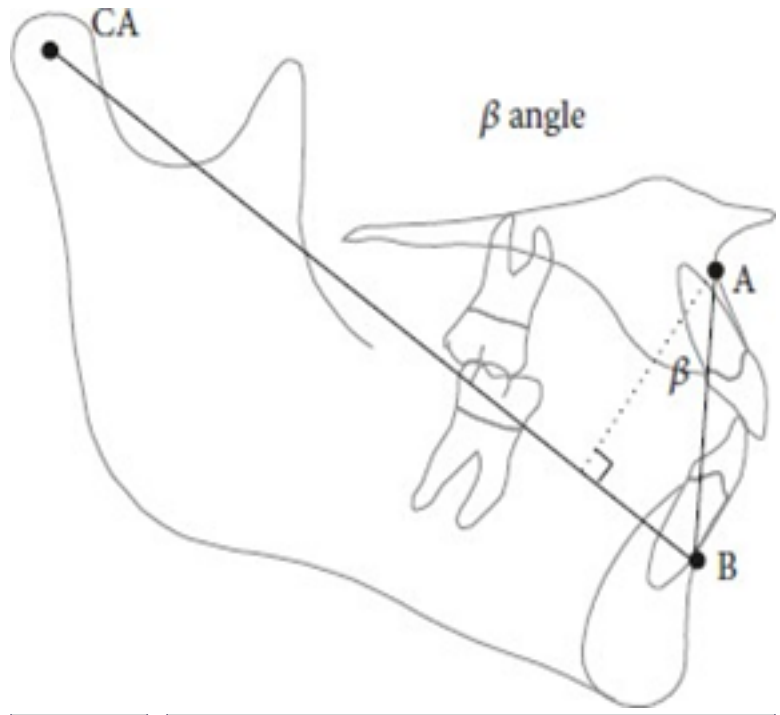

Figure 1C: MMBP-Wits

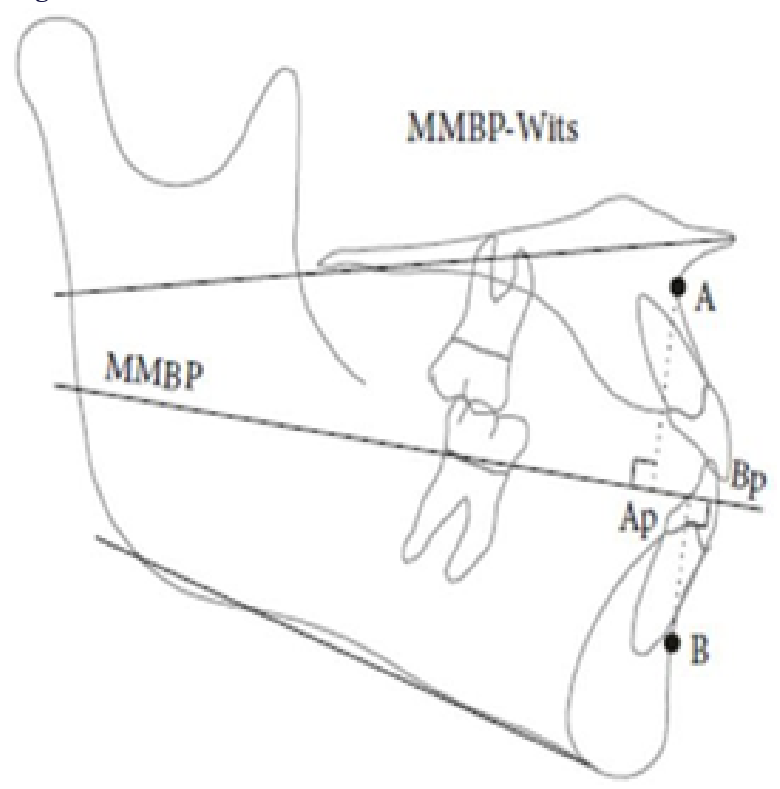

Method Error and Statistical Analysis:

Data analysis was completed using SPSS software (SPSS Inc. Chicago, Illinois, USA). Descriptive statistics for each parameter consisted of Minimum, Maximum, Mean, Median and Standard Deviation (SD). The association between SNA, SN/MP, SN/PP and NSBa angles (explanatory variables) with each ANB angle, $\beta$ angle, and MMBP-Wits (dependent variables) were assessed by backward multiple linear regression models. The cut-off levels of significance that had been used for entry and removal were 0.01 and 0.05 , respectively. Likewise, the final models, i.e. regression equations have been used to estimate the floating norms for MMBP Wits and $\beta$-angle, based on the significantly independent variables [11]. The p-value $<$ 0.05 has been considered to be statistically important.

\section{RESULTS}

Errors for angular measurements ranged from $0.5^{\circ}$ (ANB angle) to $1.2^{\circ}$ (SN/MP angle). Errors for the MMBP-Wits were of $0.6 \mathrm{~mm}$. Table 1 shows the Descriptive statistics for every parameter analyzed. Outcomes on the backward multiple linear regressions are briefed in Table 2. All the three cephalometric parameters of antero-posterior maxillary-mandibular relationship had important correlation with the explanatory variables. $\beta 2$ was ranging from 0.210 to 0.181 for the ANB angle and MMBP-Wits, correspondingly. All the explanatory variables were considerably associated with the ANB angle. Specifically, NSBa, SN/MP and the SNA angles were positively related with the ANB angle, whereas the SN/PP angle was inversely related. On the other hand, both the MMBP-Wits and $\beta$-angle were considerably associated with SN/MP and SNA angles. These explanatory variables showed positive and inverse association with the $\beta$ - angle and MMBP-Wits, respectively. Regression equations were thus derived for the MMBPWits and the $\beta$-angle and as follows: MMBP-Wits $=-0.319 \cdot$ SNA angle $-0.217 \cdot \mathrm{SN} / \mathrm{MP}$ angle +30.743 , and $\beta$-angle $=0.436 \cdot \mathrm{SNA}$ angle + $0.272 \cdot \mathrm{SN} / \mathrm{MP}$ angle -13.525 . Based on these equations, floating norms were found for each parameter according to the variations of SN/MP and SNA angles as reported in Table 3.

Table 1: Descriptive statistics for the indexes of sagittal jaw relationship and other cephalometric

\begin{tabular}{|c|c|c|c|c|}
\hline Promeler & Mean $\$ 50$ & Medon & Minimum & Moinum \\
\hline \multicolumn{5}{|c|}{ 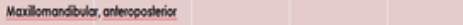 } \\
\hline ANB!" & $3.12 \div 13$ & 3 & 0.0 & 60 \\
\hline Porde it & $31.1 \pm 2.15$ & 31 & 218 & 388 \\
\hline 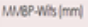 & $.18 \pm 24$ & .1.5 & 9.2 & 39 \\
\hline \multicolumn{5}{|c|}{ 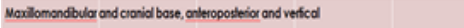 } \\
\hline SNAande $M$ & $209 \pm 205$ & 81 & $n$ & $8 \%$ \\
\hline SNPP orde li & $7 A \pm 115$ & 6 & 0.8 & 1755 \\
\hline SNMP ardo il & $32.2 \pm 3.1$ & 31 & a.s & 4.1. \\
\hline NBboande if & $1309 \pm 5.1$ & 130 & 1205 & 1002 \\
\hline
\end{tabular}

parameters $(\beta=100)$. 
Table 2: Results of the backward multiple linear regressions for the indexes of sagittal jaw relationship with each explanatory variable $(\eta=100)$.

\begin{tabular}{|l|l|l|}
\hline Explanatory variable & $\boldsymbol{\beta}$ (SE) & $\boldsymbol{t}$ \\
\hline $\begin{array}{l}\text { Model 1: dependent variable ANB } \\
\text { angle, } R 2=0.210\end{array}$ & & \\
\hline SNA angle & $0.173(0.038)$ & 4.328 \\
\hline SN/PP angle & $-0.015(0.005)$ & 2.382 \\
\hline SN/MP angle & $0.107(0.025)$ & 3.884 \\
\hline NSBa angle & $0.054(0.021)$ & 2.390 \\
\hline Explanctory variable & $\boldsymbol{\beta}($ SE) & $\boldsymbol{t}$ \\
\hline
\end{tabular}

Model 2: dependent variable $\beta$ angle,

$R 2=0.187$

\section{SNA angle}

SN/MP angle

Explanalory variable

$\beta$ (SE)

\section{$\boldsymbol{t}$}

Model 3 : dependent variable

MMBP-Wits, $R 2=0.181$

SNA angle

$-0.319(0.069) \quad-4.532$

SNMP angle

$-0.217(0.049)-4.264$

Table 3: Floating norms for the $\beta$ angle and MMBP-Wits according to the SNA and SN/MP angles $(\eta=100)$.

\begin{tabular}{|c|c|c|c|c|c|c|c|c|c|c|}
\hline Promeler & $\begin{array}{l}\text { SN/MP } \\
\text { ande(t) }\end{array}$ & 74 & 76 & 78 & 80 & 82 & 84 & 86 & 88 & 90 \\
\hline & 24 & 8 & $\alpha$ & $v$ & 28 & $\gamma$ & 30 & 31 & $n$ & $n$ \\
\hline & 2 & 27 & 27 & $x$ & $\vartheta$ & 30 & 31 & 3 & 3 & 34 \\
\hline \multirow[t]{4}{*}{ Borge|| } & $\eta$ & $\gamma$ & $y$ & 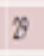 & 30 & 31 & $n$ & 30 & 34 & 35 \\
\hline & 36 & $y$ & 30 & 31 & 31 & 3 & 30 & 34 & 35 & 36 \\
\hline & 40 & 30 & 3 & 3 & 3 & 33 & 34 & 3 & 36 & $y$ \\
\hline & 44 & 31 & $?$ & 33 & 34 & 34 & 35 & 36 & 3 & 3 \\
\hline \multirow[t]{3}{*}{ Promele } & $\begin{array}{l}\text { SNINP } \\
\text { onde(') }\end{array}$ & 74 & 76 & 78 & 80 & 82 & 84 & 85 & 88 & 90 \\
\hline & 24 & 2 & 1 & 0 & 0 & 1 & 1 & 2 & 3 & 3 \\
\hline & 2 & 1 & 0 & 0 & .1 & -2 & 2 & 3 & 4 & 4 \\
\hline \multirow{4}{*}{$\begin{array}{l}\text { Migents } \\
\mid \mathrm{mel}\end{array}$} & 3 & 0 & -1 & -1 & 2 & 3 & 3 & 4 & 5 & 5 \\
\hline & $x$ & .1 & 2 & -2 & 3 & 3 & 4 & 5 & 5 & 6 \\
\hline & 0 & -2 & -2 & 3 & 4 & 4 & 5 & 6 & 6 & .7 \\
\hline & 4 & 3 & 3 & 4 & .5 & .5 & 6 & 6 & .7 & 8 \\
\hline
\end{tabular}

\section{DISCUSSION}

With the utilization of multivariate models, the floating norms for two of the three indices that have been previously mentioned have earlier been measured in Caucasian subjects [1]. With an aim to identify a more suitable index for sagittal jaw relationship, a number of analyses had been introduced namely; W angle [19], Pi analysis [18] and AXB angle [6].

The SNA angle and SN/MP angles were found to have a significant role in distorting the indices of jaw relationship among the cephalometric parameters investigated. However, these indices are based on anatomical landmarks and geometric relationships and can be affected by facial divergence and a variety of morphological localization of the landmarks. $[4,6,7,20,21]$.

All the parameters are based on standard mean values which are obtained from subjects with a near ideal and well-balanced occlusion. It has been shown by Solow [22] that there may be a correlation between a certain pattern and cephalometric skeletal variables. By this it becomes evident that, although all the cephalometric variables of a patient lie beyond or at one standard deviation, they may still be recognized even if they have only a definite relation with each other. Also, these indices are said to be geometrically sensitive and may have the tendency to produce false results. A morphological feature such as the facial divergence has been found to drastically affect the consistency of cephalometric indices of the skeletal class. [6,7]

Since most of the earlier research on measuring the facial divergence had been done with ANB angle and FOP-Wits only, there is an increased need to investigate on the "if and how" of any morphological variable such as facial divergence, would affect their dependability in terms of sagittal relationship of the jaws with the use of other angles as well. Further shortcomings of the previous investigations have stated that either only subjects with the normal divergence were analyzed [9], or a bivariate correlation analysis was used to study the harmony between the two skeletal class indices [20].

The findings for normal ANB angle values (3.12 \pm 1.3$)$ and $\beta$ angle values $(31.1 \pm 2.15)$ obtained for skeletal Class I subjects in the present sample (Table 1) are very close to those of numerous previously published studies [3, 9]. However, normal values for the MMBP-Wits seen here in were of $-1.8 \pm 2.4$, whereas the mean normal value has been found to be $-4 \mathrm{~mm}[10]$. Nevertheless, this variance would have slight clinical importance, and this variance in the sample under investigation may be a justification.

In the current study, the degree of association between the indices of sagittal jaw relationship (ANB, $\beta$ angle, and MMBP-Wits) and the angles SN/MP, SNA, NSBa and SN/PP has been calculated (Table 2). Angular parameters have been selected for the analyses without being affected by magnification. Thus, that would make the subsequent results more reproducible. Furthermore, the incorporated sample size in the present study was of large in size.

The findings showed that the ANB angle is the parameter that was most affected. In particular, even the NSBa angle may have an effect of distortion of this index of sagittal jaw relationship (Table 2). This research is consistent with previous studies [5-7, 23, 24] and suggests that the ANB angle should not be used in patients with significant deviation from the norm, in terms of facial divergence or maxillary protrusion. Whereas, the analyzed cephalometric parameters showed that the MMBP-Wits and the $\beta$ angle were to be less affected, and hence instinctive floating norms could be obtained (Table 3 ).

Using the SN/PP, SNA, SN/MP, and NSBa as independent variables and the ANB angle as a dependent variable, resulting $\beta 2$ is equal to 0.210 ; i.e., all the four cephalometric parameters effect the ANB angle variability for a total of about $21 \%$ of the value. $\beta 2$ for the MMBPWits and $\beta$ angle and was 0.181 and 0.187 , respectively. Consequently, the SN/MP and the SNA angle alone would be a reason for about 18 $19 \%$ of their variability (Table 2 ).

The regression equations for MMBP-Wits and $\beta$ angle were as follows: MMBP-Wits $=-0.319 \cdot$ SNA angle $-0.217 \cdot \mathrm{SN} / \mathrm{MP}$ angle +30.743 , and $\beta$ angle $=0.436 \cdot$ SNA angle $+0.272 \cdot$ SN/MP angle -13.525 . Floating norms have been acquired for most common situations of SNA angle (from $74^{\circ}$ to $90^{\circ}$ ) and SN/MP angle (from $24^{\circ}$ to $44^{\circ}$ ) (Table 3) by the application of such equations. By modifying the MMBP-Wits and $\beta$ angle for the maxillary protrusion and facial divergence with the help of such floating norms, it is likely to analyze the sagittal jaw relationship.

Cephalometric diagnosis has been used for the application of separate normal values derived from a statistical population. However, the breakthrough of an impact of this cephalometric diagnosis on the indices of antero-posterior sagittal discrepancy, deriving from divergence, made this concept outdated.

A small number of subjects can have a class I skeletal relationship with a harmonic profile in spite of deviating from the norm values. In situations like these, it is said to be beneficial to replace cephalometric 
normal values with mean values derived from a population sample with floating norms based on the relationship between appropriate cephalometric variables $[11,12,15]$. Nonetheless, since floating norms are acquired from regression equations, the accuracy of the diagnosis would entirely depend on the standard errors that have been retrieved from the equations.

In order to gain a more accurate diagnosis from the data collected in this analysis, clinicians should use the less biased index to calculate the sagittal jaw relation. Without a doubt, it is possible to derive individualized values for each patient using the floating norms for angles SN / MP and SNA (Table 3). Similarly, considering the standard deviations from the present study (Table1), the corresponding intervals of such individualized norms can be obtained. If the value belongs to the mean value and standard deviation given interval, the subject can be diagnosed as having normal sagittal jaw relationship, i.e. skeletal Class I malocclusion. Values outside such intervals would thus be suggestive of skeletal Class II or Class III malocclusion.

\section{CONCLUSIONS}

(I) ANB angle is prone to have increased geometrical distortion when compared to $\beta$ angle and MMBP-Wits

(ii) To individualize the reference values for both the MMBP-Wits and the $\beta$ angle, floating norms were given.

\section{Conflicts of Interest}

The authors declare that there are no conflicts of interest regarding the publication of this paper.

\section{REFERENCES}

[1] Giuseppe Perinetti, Michele Ceschi, Alessandro Scalia, and Luca Contardo. (2018), "Cephalometric Floating Norms for the Angle and MMBP-Wits." Hindawi BioMed Research International Volume 2018.

[2] R. A. Riedel. (1952)" The relation of maxillary structures to cranium in malocclusion and in normal occlusion" The Angle Orthodontist vol. $22 \mathrm{pp} .142-145$.

[3] C. C. Steiner. (1953) "Cephalometrics for you and me" American Journal of Orthodontics and Dentofacial Orthopedics vol. 39 no. 10 pp $729-755$

[4] A. Jacobson. (1975) "The "Wits" appraisal of jaw disharmony." American Journal of Orthodontics and Dentofacial Orthopedics.vol. 67 no. 2 pp 125-138.

[5] S. J"arvinen. (1985) "An analysis of the variation of the ANB angle: A statistical appraisal." American Journal of Orthodontics and Dentofacial Orthopedics.vol. 87 no. 2 pp. $144-146$.

[6] R. S. Freeman. (1981) "Adjusting A-N-B angles to reflect the effect of maxillary position."The Angle Orthodontist, vol. 51 no. 2 pp.162-171.

[7] W.Hussels and R. S.Nanda.(1987) "Clinical application of a method to correct angle ANB for geometric effects." American Journal of Orthodontics and Dentofacial Orthopedics, vol. 92 , no. 6, pp.506-510

[8] R. Rushton, A. M. Cohen, and A. D. Linney (1991)" The relationship and reproducibility of angle ANB and the Wits appraisal." British Journal of Orthodontics, vol. 18, no. 3, pp. 225-231.

[9] C. Y. Baik and M. Ververidou. (2004) "A new approach of assessing sagittal discrepancies: The Beta angle. American Journal of Orthodontics and Dentofacial Orthopedics", vol. 126, no. 1, pp.100-105.

[10] J.Hall-Scott. (1994) "The maxillary-mandibular planes angle (MMo) bisector: A new reference plane for anteroposterior measurement of the dental bases." American Journal of Orthodontics and Dentofacial Orthopedics, vol. 105, no. 6, pp. 583-591.

[11] S.J"arvinen.(1986) "Floating norms for the ANB angle as guidance for clinical considerations."American Journal of Orthodontics and Dentofacial Orthopedics. vol. 90, no. 5 , pp. $383-387$

[12] L. Franchi, T. Baccetti, and J.A. McNamaraJr. (1998)"Cephalometric floating norms for North American adults.”'The Angle Orthodontist, vol.68, no. 6, pp. 497-502.

[13] A. Hasund and O. E. B" oe. (1980) "Floating norms as guidance for the position of the lower incisors." The Angle Orthodontist, vol. 50, no. 3, pp. 165-168.

[14] D. Segner.(1989) Floating norms as a means to describe individual skeletal patterns. European Journal of Orthodontics, vol. 11, no.3, pp. 214-220.

[15] I. Tollaro, T. Baccetti, and L. Franchi.(1996) "Floating norms for the assessment of craniofacial pattern in the deciduous dentition."'European Journal of Orthodontics vol. 18 , no. 4 , pp. $359-365$

[16] G. Perinetti, C. Cordella, F. Pellegrini, and P. Esposito.(2008) "The prevalence of malocclusal traits and their correlations in mixed dentition children: results from the Italian OHSAR Survey." Oral Health \& Preventive Dentistry, vol. 6, no. 2, pp. 119-129.

[17] G. Perinetti,(2016) "StaTips Part II: Assessment of the repeatability of measurements for continuous data." South European Journal of Orthodontics and Dentofacial Research, vol. 3, no. 2, pp. 33-34

[18] S. Kumar et al. (2012) "An evaluation of The Pi analysis in the assessment of anteroposterior jaw relationship." Journal of Orthodontics, vol.39, no. 4, pp. 262-269.

[19] W. A. Bhad, S. Nayak, and U. H. Doshi.( 2013) "A new approach of assessing sagittal dysplasia: The W.angle." European Journal of Orthodontics, vol. 35, no. 1, pp. 66-70.

[20] H. Oktay. (1991) "A comparison of ANB, WITS, AF-BF, and APDI measurements." American Journal of Orthodontics and Dentofacial Orthopedics, vol. 99, no. 2, pp. $122-128$.

[21] C. M. Taylor.(1969) "Changes in the relationship of nasion, point A,and point B and the effect upon ANB." American Journal of Orthodontics and Dentofacial Orthopedics, vol. 56, no. 2, pp.143-163.

[22] B. Solow,(1966) "The pattern of craniofacial associations - a morphological and B. Solow, (1966) "The pattern of craniofacial associations - a morphological and
methodological correlation and factor analysis study on young male adults." American methodological correlation and factor analysis study on young male adults." American Journal of Orthodontics and Dentofacial Orthopedics vol. 24,46.

[23] K. Hurmerinta, A. Rahkamo, and K.Haavikko.(1997)"Comparison between cephalometric classification methods for sagittal jaw relationships." European Journal of Oral Sciences, vol. 105, no. 3,pp. 221-227.

[24] S. Rotberg, N. Fried, J. Kane, and E. Shapiro.(1980)"Predicting the"Wits" appraisal from the ANB angle." American Journal of Orthodontics and Dentofacial Orthopedics, vol. 77, no. 6, pp.636-642. 